\title{
THIERRY LAURENT
}

Centre de Recherche de I'ICES

\section{Prosper Mérimée et la Pologne}

D rosper Mérimée, esprit curieux, passionné par les cultures étrangères, observateur attentif mais souvent partial de la politique internationale, s'est intéressé à la Pologne même si c'est dans une bien moindre mesure qu'à la civilisation russe et avec un manque d'empathie qui peut surprendre. La lecture de sa correspondance ${ }^{1}$ nous en donne bien des exemples et révèle une hostilité qui allait croissant au fil des décennies².

L'insurrection populaire, nationale et antirusse des années 1830 et 1831 est le premier événement à propos duquel il montre son indifférence pour la Pologne, contrairement à Musset ou au jeune Flaubert qui sympathisent d'emblée avec les révoltés. Tout libéral et progressiste qu'était Mérimée, il se méfiait des processus révolutionnaires ou de ce qu'il croyait tels, et, séjournant alors en Espagne, il n'avait ni participé aux émeutes parisiennes de juillet 1830 qui avaient contraint Charles $X$ à abdiquer ni fait part d'un grand enthousiasme à l'annonce de la

\footnotetext{
${ }^{1} \mathrm{P}$. Mérimée, Correspondance générale, M. Parturier, P. Josserand, J. Mallion (dir.), Paris, Le Divan, 1941-1947, t. 1-6 ; Toulouse, Privat, 1953-1964, t. 7-17 ; $C G$, tome, pagination par la suite.

2 Pour la rédaction de cet article, je suis redevable envers Zygmunt Markiewicz (1909-1991), historien des littératures française et polonaise, qui, il y a presque soixante-dix ans, consacrait déjà une étude savante à ce sujet : Z. Markiewicz, "La Pologne dans l'œuvre et la vie de Mérimée ", [dans :] Revue de Littérature comparée, avril-juin 1953, vingt-septième année, $n^{\circ} 2$, p. 148-159. Je me permettrai de lui emprunter beaucoup d'arguments et exemples. Et je remercie vivement Antonia Fonyi, présidente de la Société Mérimée, pour ses informations, ses conseils et ses corrections.
} 
nouvelle. Alors que son ami Édouard Grasset voulait se rendre en Pologne avec un bateau transportant des armes pour les insurgés, Mérimée, qui servait d'intermédiaire entre lui et Marie de Neuville dans leurs querelles d'amoureux, relate avec beaucoup d'ironie la réaction de la jeune fille à la nouvelle de ce départ, qui d'ailleurs n'eut pas lieu :

C'est pour cette cause qui ennoblit ceux qui la défendent qu'il a senti se réveiller en lui une noble ambition et comment ne pas réussir, ne pas acquérir de la gloire avec tous ces héros ! Dieu le veuille ! Dieu protège ceux qui deviennent polonais ! (CG, 1, 128-129)

Au cours des années suivantes, il s'en prendra à plusieurs reprises à des personnalités polonaises en exil à Paris. Par exemple, il montre très tôt son antipathie pour l'ambitieux Alexandre Walewski, fils de Napoléon ler et de Marie Walewska, qui fait une carrière littéraire et politique en France et deviendra ministre de Napoléon III3 ; il ironise sur la bisexualité du comte Ignace Gurowski qui, amant du marquis de Custine, a épousé une infante d'Espagne (CG, 3, 56-57). À l'égard de l'écrivain romantique Adam Mickiewicz, ses sentiments sont partagés : il admire certes son poème Pan Tadeusz (Messire Thadée), écrit et publié à Paris en 1834, mis à I'honneur par George Sand, et qui paraîtra en français en 1859 dans la traduction de Christien Ostrowski, mais il considère l'homme comme un exalté peu recommandable ! Le 23 mars 1844, il écrit à Mme de Montijo :

II y a quatre jours M. Mickiewicz, un Polonais qui fait un cours de slave au Collège de France, au lieu de parler de déclinaisons et de conjugaisons s'avisa de faire une tartine du mysticisme le plus abstrus, un véritable sermon dans le genre de Jérémie. Puis il s'interrompit tout-à-coup pour demander à son auditoire s'il avait ses sympathies. "Oui, vous les avez ", s'écrièrent cent jeunes gens à barbe plus ou moins mal peignée ; et une femme s'élançant vers sa chaire, "Je veux mourir sur la croix ! " et pour commencer elle tombe à la renverse et a une attaque de nerfs. (CG, 4, 67-68)

$\overline{3 \text { Voir par exemple : } C G}, 2,301$ et $C G, 3,462$. 
S'adressant encore à Mme de Montijo, cette fois à propos des tentatives d'insurrection qui secouent la Pologne vers 1846 , il emploie un ton léger et ironique qui cadre mal avec la gravité des événements racontés :

Les empereurs de Russie et d'Autriche vont encore se donner le plaisir de faire fusiller ou pendre un certain nombre de Polonais. On dit que cela ne les fera pas aimer davantage par leurs sujets. (CG, 4, 429)

En même temps, c'est un homme généreux qui aide des exilés. Dans sa correspondance, entre 1833 et 1849 , on trouve plusieurs allusions au sujet des émigrés polonais et tout spécialement d'un certain Joseph Louis Schlesinger, diplômé de l'université de Vienne, médecin réfugié : à deux reprises, il se sert de ses influences pour lui rendre possible l'exercice de sa profession ${ }^{4}$; il rend également service à l'un des Potocki, grande famille aristocratique polonaise ${ }^{5}$. Lorsque, vers 1850 , la préparation de son livre sur les faux Démétrius l'oblige à faire des recherches à la Bibliothèque polonaise de Paris ${ }^{6}$, il se lie d'amitié avec le général Ladislas Zamoyski (héros de la révolte de 1830) qui I'y a introduit, le prince Adam Czartoryski (ancien chef du gouvernement provisoire) qui l'autorise à emprunter des documents, et Karol Sinkiewicz, conservateur et savant éminent. Ultérieurement, Mérimée suggérera à la Société historique et littéraire polonaise (fondée en 1832) de faire des démarches pour avoir un statut juridique plus favorable ; finalement, en 1866, la Société deviendra un établissement d'utilité publique par décret de Napoléon III et en sera reconnaissante à Mérimée qui, de son côté,

\footnotetext{
${ }^{4}$ Z. Markiewicz, " La Pologne dans l'œuvre et la vie de Mérimée », op. cit., p. 155.

5 Ibidem.

6 II s'y documentera sur l'histoire de Sigismond III Vasa, roi de Pologne (1587-1632), celle du faux Dimitri II (tsar entre 1605 et 1606), ainsi que sur l'audience de Vassili IV Chouïski (tsar de 1606 à 1610) devant la Diète. II y admirera une gravure publiée en Pologne en 1606 représentant le premier faux Démétrius.
} 
parlera toujours très positivement de ses bons rapports avec les membres de cette institution.

En décembre 1852, l'écrivain publie dans La Revue des Deux Mondes son étude intitulée Le faux Démétrius, scènes dramatiques ${ }^{7}$. II y a d'autre part, en 1853, chez éditeurs Michel Lévy frères, l'ouvrage historique Épisode de l'histoire de la Russie. Les Faux Démétrius (pluriel cette fois) ${ }^{8}$. L'idée lui est venue de traiter ce sujet en lisant, en version originale, Boris Godounov d'Alexandre Pouchkine datant de $1831^{9}$. Il a utilisé de nombreuses sources plutôt sérieuses bien que pas toujours objectives, comme l'Histoire de l'État russe de Nikolaï Karamzine ${ }^{10}$ sur laquelle avait déjà travaillé Pouchkine, L'Estat de l'Empire de Russie et du Grand-Duché de Moscovie, récit du capitaine Margeret, autrefois au service de Boris et de Démétrius, les Mémoires contemporains sur le faux Démétrius traduits par Nicolas Oustrialov ${ }^{11}$, les œuvres de Platon Pavlov (auteur d'un mémoire en 1850 sur le règne de Boris Godounov), de Nikolaï Kostomarov (grand historien russo-ukrainien du XIX siècle, orthodoxe convaincu) ainsi que quelques

\footnotetext{
7 Dans sa biographie de l'écrivain, Xavier Darcos relate la genèse du projet et présente les sources utilisées. Cf. X. Darcos, Mérimée, Paris, Flammarion, 2009, p. 401-402.

8 Jean-Louis Backès et Pierre Gonneau ont donné les explications sur ce sujet-là. Cf. Prosper Mérimée, Histoire de Russie, I. Les Faux Démétrius, textes établis, présentés et annotés par Jean-Louis Backès et Pierre Gonneau, [dans :] Idem, CEuvres complètes, A. Fonyi (dir.), Paris, Honoré Champion, 2012, section III, "Histoire ", t. 3.

9 Voir sa lettre à Madame de Lagrené, sa professeure de russe, du 31 août 1849, que nous signale Robert Baschet dans Du Romantisme au Second Empire, Mérimée (1803-1870), Paris, Nouvelles Éditions Latines, 1959, p. 162. ${ }^{10} \mathrm{Grand}$ historien russe qui dissuada Alexandre ler de restaurer le royaume de Pologne ; Mérimée avait lu dans la traduction française le passage concernant les éléments bien connus du règne de Boris Godounov.

11 Les cinq tomes lui ont été rapportés de Saint-Pétersbourg par Serge Sobolevski, (bibliophile que Mérimée connaissait depuis 1829, rencontré chez Mme Ancelot, et avec qui il conservera d'étroites relations) ; il en donne un compte rendu dans deux articles du Journal des Savants : P. Mérimée, "Mémoires contemporains sur le faux Démétrius ", [dans :] Journal des Savants, février 1852, p. 88-100 ; mars 1852, p. 159-174.
} 
mémoires contemporains polonais en latin. Mérimée indique à Serge Sobolevski le 14 avril 1852 :

Après avoir bien étudié mon affaire, l'idée m'était venue d'écrire l'histoire comme elle a dû se passer, affirmant au lieu de présenter une hypothèse. J'ai fait sur ce thème quelques scènes qui m'ont conduit jusqu'en Pologne. Là, rencontrant Pouchkine, j'ai abandonné mon projet (grand dommage) et j'ai écrit une vulgaire histoire. ${ }^{12}$

Dans les quelque trois cents pages d'Épisode de l'histoire de Russie. Les Faux Démétrius, il s'efforce de démontrer que le premier faux Démétrius ${ }^{13}$, Dimitri II (1605-1606), tsar réformateur ayant prétendu être le fils d'Ivan IV, soutenu par les Polonais, était un cosaque originaire d'une région soumise à la Pologne où l'on parlait le polonais et le russe, sans doute l'Ukraine ; il revient évidemment sur la question de savoir si Boris Godounov n'aurait pas tué le vrai Démétrius ; il parle encore du second faux Démétrius qui, lui, se disait fils du tsar Fédor II et tenta vainement d'accéder au trône de Russie avec, là encore, le soutien initial des Polonais. Au-delà de l'enquête, ces histoires d'aventures quasiment romanesques permettent à Mérimée de retrouver ses thèmes de prédilection : mystifications et magie, cruautés et massacres, tragi-comédie. Son livre eut un certain succès, beaucoup de lecteurs ayant été sensibles à l'art du récit. Sur le plan strictement historique, il y a sans doute à redire d'autant que le parti pris de

12 P. Mérimée, Lettre à Serge Sobolevski, le 14 avril 1852, [cité d'après :] R. Baschet, Du Romantisme au Second Empire..., op. cit., p. 162.

${ }^{13}$ Rappelons, pour mémoire, que ce personnage illuminé et grand guerrier, soutenu par Sigismond III de Pologne, qui avait été protégé en Lituanie par le prince Adam Viszniowiecki, réussit à entrer à Moscou à la tête d'une armée, à la mort de Boris Godounov, pour s'y faire couronner tsar sous le nom de Dimitri II en mai 1606 ; il épousera Marine, princesse polonaise catholique. Un an plus tard, il sera massacré par des tenants de la tradition orthodoxe, en même temps que beaucoup de Polonais catholiques vivant à Moscou. Vassili IV lui succède. II y eut quelque temps plus tard un autre prétendant au trône de Russie, probablement cosaque, qui se disait fils du tsar Fédor ; il créa sa cour à Touchino, se proclamant tsar, épousant Marine, la veuve de Dimitri II, sans doute aidé par Sigismond III avant que ce dernier ne fasse des tractations avec Vassili IV ; il mourra assassiné. 
l'auteur est d'insister sur le fait que la Pologne tirait les ficelles de tous ces événements en vue d'obliger la Russie à se convertir au catholicisme et que son roi Sigismond III n'était qu'un ambitieux maladroit. D'emblée d'ailleurs, les Polonais sont présentés dans l'Histoire comme belliqueux, inconstants et désunis. En outre, Mérimée le voltairien malmène parfois la réalité en accusant la Pologne catholique un peu à la légère :

Ses rois, dans leurs démêlés avec la Russie, étaient animés autant par la ferveur religieuse que par l'ambition d'augmenter leur empire. Souvent ils proclamèrent le projet d'extirper le schisme d'Orient, et, sacrifiant leurs intérêts politiques à leur zèle de conversion, ils obligèrent les chrétiens du rite grec à se jeter dans les bras de la Russie. C'est de la sorte qu'ils s'aliénèrent les Lithuaniens et les peuples guerriers de l'Ukraine. ${ }^{14}$

On pourrait donner bien d'autres exemples des " piques » que lance l'écrivain à l'encontre de la Pologne de jadis. En tout cas, le sujet le passionne tellement qu'il s'en inspire pour écrire encore en 1852 une variante de l'histoire d'un des deux faux Démétrius, qu'il recueillera en volume sous le titre Les Débuts d'un aventurier, roman dialogué qui se passe en partie en Pologne et qu'appréciera particulièrement Hippolyte Taine.

Dans " Les Cosaques de l'Ukraine et leurs derniers atamans ", qui parut dans le Moniteur universel du 21-23 juin 1854, Mérimée excelle à décrire cette communauté un peu "sauvage " par sa manière de vivre et son caractère guerrier et cruel. C'est aussi l'occasion pour lui de reparler de la politique étrangère et militaire de la Pologne, cette fois au temps du roi Jean II Casimir (16481668) qui ne parvint pas à maintenir l'intégrité de son État face aux Russes flanqués des Tatars et des Cosaques; en cause, l'attitude immature de sa noblesse, y compris lorsqu'elle a obtenu l'avantage sur le terrain militaire :

14 P. Mérimée, Épisode de l'histoire de Russie. Les Faux Démétrius, Paris, Michel Lévy frères, 1854, p. 35. 
La victoire de Berestençko fut glorieuse, mais stérile. Après avoir sabré les fuyards pendant plusieurs lieues, les nobles polonais crurent que la guerre était finie et voulurent regagner leurs châteaux. [...] Tel fut, tel devait être le triste résultat des exploits de cette noblesse polonaise si héroïque sur le champ de bataille, mais toujours et partout imprévoyante et indisciplinée. ${ }^{15}$

Dans Les Cosaques d'autrefois (1865) ${ }^{16}$, compte rendu analytique de l'ouvrage partial de l'historien Kostomarov, apologiste des cosaques et partisan de l'Ukraine russifiée, Mérimée évoque les destins tourmentés de deux personnages célèbres: Stenka Razine, cosaque du Don, qui s'opposa à l'État, aux institutions, à la noblesse, et Bogdan Chmielnicki, ennemi de la noblesse polonaise et massacreur de Juifs, militaire et homme politique engagé pour l'autonomie (relative) de l'Ukraine. Voici comment Mérimée reprend à son compte la peinture de la situation en Pologne au moment de l'élection de Jean-Casimir. Le tableau est bien noir :

Une armée vivant de pillage, des chefs sans expérience, des paysans abrutis par la misère et prêts à suivre quiconque porterait le fer et le feu chez leurs maîtres, une assemblée impatiente de toute supériorité, des délibérations tumultueuses, les suffrages obtenus par la terreur ou la corruption, tel était le spectacle que Varsovie présentait à cette époque [...]. ${ }^{17}$

De même, les conclusions de Mérimée exagèrent le rôle et la valeur du chef militaire des cosaques, Chmielnicki, présenté comme un grand homme politique :

Si Chmielnicki eût vécu sous un roi Batory [roi de Pologne de 1576 à 1586], il l'eût sans doute aidé à réformer l'absurde constitution de la

${ }_{15}$ P. Mérimée, Mélanges historiques et littéraires, Paris, Michel Lévy frères, 1853, p. 80-81.

16 P. Mérimée, Les Cosaques d'autrefois, Paris, Michel Lévy frères, 1865. Le livre contient deux récits : Bogdan Chmielnicki (six articles publiés d'abord dans le Journal des Savants de 1863 : P. Mérimée, "Bogdan Chmielnicki ", [dans :] Journal des Savants, janvier 1863, p. 5-25 ; février 1863, p. 77-95 ; mars 1863 , p. $153-175$; mai 1863 , p. $277-297$; juin 1863, p. 362-383 ; juillet 1863, p. 432-453) et Stenka Razin (article intitulé initialement " La révolte de Stenka Razin ": P. Mérimée, "La révolte de Stenka Razin ", [dans :] Journal des Savants, juillet 1861, p. 389-420).

17 P. Mérimée, Les Cosaques d'autrefois, op. cit., p. 21. 
Pologne et à substituer une monarchie forte à l'aristocratie anarchique et fatale à ce pays. Malheureusement il ne trouva que des princes inconstants et légers, et ils ne virent qu'un rebelle dans l'homme qui pouvait être leur plus utile instrument. Bien qu'il ménageât la Pologne, qui deux fois fut à ses pieds, Chmielnicki lui porta le coup le plus funeste en lui ôtant l'Ukraine et en introduisant, pour ainsi dire, les Moscovites dans le secret de la faiblesse de la république. Dans un jour de colère, il prépara son démembrement. ${ }^{18}$

Aux approches de l'insurrection polonaise de 1863, l'attitude de Mérimée - probablement sous l'influence de ses nombreux amis russes - devient nettement hostile. Quelques années après la fin de la guerre de Crimée, la France de Napoléon III veut la réconciliation avec la Russie d'Alexandre II mais les sentiments pro-polonais sont encore très forts parmi les intellectuels et dans la classe politique : pour ne citer qu'un exemple bien connu, le prince Napoléon, cousin de l'empereur, dit au Sénat le 18 mars : "J'ai le cœur polonais ! ". Finalement, la Russie n'appréciera pas l'attitude très ambiguë de la France et se rapprochera de l'Angleterre.

La correspondance de Mérimée avec Antonio Panizzi, (Italien réfugié en Angleterre, conservateur au British Museum, porte-parole de la diplomatie secrète de Napoléon III auprès des hommes d'État anglais), qui embrasse la période 1850-1870, outre le fait qu'elle révèle chez l'écrivain son anti-américanisme, sa haine de Garibaldi ou son admiration pour Bismarck, montre une hostilité grandissante à l'idée d'indépendance polonaise. Déjà en juillet 1859, il lui écrivait :

La Hongrie et la Bohème ne tenaient plus qu'à un fil, et les Polonais, qui sont en possession de gâter tout, pour se venger de l'indifférence de l'Europe, avaient pris une attitude qui devait nous priver de tout appui du côté de la Russie, et peut-être même l'obliger à se déclarer contre nous. $(C G, 1,55)$

18 Ibidem, p. 293-294. 
En mai 1863, la situation s'aggravant, il continuera de penser que les Polonais menacent la paix et que la France a tout intérêt diplomatiquement à se lier à la Russie :

On s'occupe toujours beaucoup, et à mon avis trop, des affaires de Pologne. Heureusement, jusqu'à présent, et j'espère que cela continuera, on s'en occupe diplomatiquement et de concert avec l'Angleterre et l'Autriche. II faut que la guerre de Crimée ait blessé la Russie plus fortement qu'on ne pensait pour qu'elle n'en ait pas encore fini avec cette révolte qui, même en tenant compte des exagérations des journaux, paraît s'étendre et s'envenimer tous les jours. (CG, 1, 320)

Dans ses lettres à d'autres amis, notamment Jenny Dacquin, il répète à l'envi que les journaux français disent n'importe quoi à propos des affaires de Pologne ou qu'il n'y a pas un mot de vrai dans les bulletins polonais. On peut être étonné que les liens qu'il a eus à Paris, notamment par l'intermédiaire de George Sand, avec de nombreux membres de l'aristocratie polonaise (par exemple la comtesse Potocka ou la comtesse Przezdziecka) n'aient pas infléchi ses positions; on peut prudemment avancer la thèse que toutes ces connaissances n'ont pas eu la durée ni le poids de ses amitiés russes, que ses préjugés anti-polonais (qui n'étaient pas rares au milieu du siècle, pensons à Balzac et sa Cousine Bette ${ }^{19}$ ) se nourrissaient simultanément - et peut-être confusément - de son anticatholicisme, de sa peur des convulsions révolutionnaires (la tentative d'assassinat d'Alexandre II à Paris en 1867 par un nationaliste polonais le révulse), d'une russophilie linguistique et littéraire grandissante après 1850, de son amour des cosaques ("Je suis Cosaque ${ }^{20}$ ), de sa

\footnotetext{
19 « Le Polonais a dans le caractère les enfantillages et l'inconstance des nations imberbes. Il possède le courage, l'esprit et la force; mais, frappés d'inconsistance, ce courage et cette force, cet esprit, n'ont ni méthode, ni esprit, car le Polonais offre une mobilité semblable à celle du vent qui règne sur cette immense plaine coupée de marécages ». $\mathrm{H}$. Balzac, La Cousine Bette, [dans :] Idem, CEuvres complètes, Paris, Houssiaux, 1874, t. 17, p. 193.

20 P. Mérimée, Lettres à une autre inconnue, Paris, Michel Lévy frères, 1875, p. 118.
} 
quasi-ignorance de la langue et de la littérature polonaises (« Je ne comprends pas un mot du polonais » $(C G, 6,76)$ ), d'un choix de lectures très orientées (comme celles des historiens russes ou ukrainiens déjà cités, des œuvres de Marko Vovtchok, femme de lettres ukrainienne qui a séjourné à Paris, (que lui a fait lire Tourguéniev), peu tendre envers les Polonais dans certains écrits, ce qui était aussi le cas de Nicolas Gogol, notamment dans son Tarass Boulba ${ }^{21}$.

À la fin de sa vie, il écrit Lokis, une nouvelle à demi fantastique qui se passe en Lituanie 22 . Ce territoire qui, au Moyen Âge, constituait le cœur du plus vaste État d’Europe (le Grand-Duché de Lithuanie) est désormais sous l'influence culturelle de la Pologne et sous la domination politique russe. Passionné par les langues rares, Mérimée s'est mis à étudier le vieux dialecte lituanien de Samogitie dont Ferdinand de Saussure expliquera qu'il est l'un des plus anciens idiomes indo-européens, assez proche du sanscrit. Pour enrichir sa documentation et donner à son récit une " couleur locale " qui dépaysera le lecteur, il se sera plongé dans des ouvrages savants concernant la République des Deux-Nations (union polonolituanienne aux XVIe et XVIIe siècles) ainsi que la géographie et la culture des régions baltes ${ }^{23}$, et il aura renoué avec certains Polonais de Paris capables de lui parler de la " province " lituanienne, tel Charles Edmond Chojecki, journaliste et homme de lettres, et ex-révolutionnaire ${ }^{24}$

\footnotetext{
${ }^{21}$ N. Gogol, Tarass Boulba, L. Viardot (trad.), Paris, Paulin, 1845.

22 Voir notre présentation de Lokis : T. Laurent, " Prosper Mérimée et la Lituanie ", [dans :] E. Aleksandravičius (dir.), Darbai ir Dienos, 2011, n 55, p. 173-181. J'y mentionne l'étude de référence due à Raymond Schmittlein en tête d'une édition de luxe de l'histoire (Schmittlein R., Lokis, la dernière nouvelle de Prosper Mérimée, Bade, Éditions Art et Science, 1949).

${ }^{23}$ Notamment C. Malte-Brun, Tableau de la Pologne ancienne et moderne, Paris, Henri Tardieu, 1807.

${ }^{24}$ Auteur de La Pologne captive et ses trois poètes : Mickiewicz, Krasinski, Słowacki : K. E. Chojecki, La Pologne captive et ses trois poètes : Mickiewicz, Krasinski, Słowacki, Leipzig, Brockhaus, 1864.
} 
(on ignore si Mérimée savait que l'impitoyable gouverneur russe de Lituanie, Mikhaïl Muraviev, en même temps qu'il faisait mettre à mort ou en prison des patriotes et des prêtres lituaniens, ordonnait l'interdiction des textes en alphabet latin et annonçait que tout serait fait pour que la langue lituanienne disparaisse). Il est à noter qu'on trouve dans Lokis, indépendamment d'allusions brèves à des spécificités lituaniennes ou polono-lituaniennes que nous n'allons pas ici énumérer ${ }^{25}$, un clin d'œil à Mickiewicz né à Vilnius, rappelons-le - et son Messire Thadée dont l'histoire se plaisait à évoquer la Lituanie ancienne : plusieurs passages en sont repris, paraphrasés ou adaptés; ainsi, la phrase du chapitre III " Je vous mène, Monsieur le professeur, dans une forêt où, à cette heure, existe florissant l'empire des bêtes, la matecznik, la grande fabrique des êtres ${ }^{26}$ répète, avec le même vocabulaire, une phrase du texte polonais. C'est également dans cette œuvre que Mérimée puise les éléments de la scène où le comte explique à son hôte qu'il existe une société animale organisée selon les règles politiques humaines. II n'est d'ailleurs pas exclu qu'il y ait quelque intention parodique à découvrir dans cette intertextualité.

Voici donc présentés, de façon succincte, quelques éléments biographiques et bibliographiques qui montrent que Mérimée, tout en étant un héritier des Lumières, un esprit positiviste, un érudit confirmé, n'est jamais parvenu à bien comprendre les particularismes polonais ni les raisons anciennes et contemporaines des revendications nationales d'un pays malmené par l'Histoire au cours du XIXe siècle et pourtant lié à la France depuis longtemps. Son admiration pour la Russie, confortée par son excellente connaissance de la langue de Pouchkine et ses lectures d'historiens pas toujours impartiaux, ainsi que sa peur des

${ }^{25}$ Cf. T. Laurent, " Prosper Mérimée et la Lituanie », op. cit.

${ }^{26}$ P. Mérimée, Romans et Nouvelles, H. Martineau (dir.), Paris, Gallimard, 1951, p. 732. 
convulsions révolutionnaires, expliquent largement des prises de position politiques sans nuances, des préjugés éculés, une insensibilité aux malheurs des Polonais, qui peuvent paraître aujourd'hui étonnants, voire critiquables. En déduire pour autant qu'il fut un ennemi de la Pologne serait cependant exagéré, compte tenu de son soutien amical à de nombreux exilés installés en France ainsi qu'à la Société historique et littéraire polonaise de Paris. Une étude serait à mener à propos d'une telle ambivalence d'une part dans l'image du peuple polonais, d'autre part face à la question polonaise, chez différents auteurs français de l'époque. 


\section{bibliographie}

Baschet R., Du Romantisme au Second Empire, Mérimée (1803-1870), Paris, Nouvelles Éditions Latines, 1959.

Darcos X., Prosper Mérimée, Paris, Flammarion, 1998.

Laurent T., "Prosper Mérimée et la Lituanie ", [dans :] E. Aleksandravičius (dir.), Darbai ir Dienos, 2011, n 55.

Markiewicz Z., "La Pologne dans l'œuvre et la vie de Mérimée », [dans :] Revue de Littérature comparée, avril-juin 1953, vingt-septième année, n 2. Mérimée P., Correspondance générale, M. Parturier, P. Josserand, J. Mallion (dir.), Paris, Le Divan, 1941-1947, t. 1-6 ; Toulouse, Privat, 1953-1964, t. 7-17. Mérimée P., Les Cosaques d'autrefois, Paris, Michel Lévy frères, 1865.

Mérimée P., " Histoire de Russie, Les Faux Démétrius ", [dans :] Idem, CEuvres complètes, A. Fonyi (dir.), Paris, Honoré Champion, 2012, section III, " Histoire ", t. 3.

Mérimée P., Mélanges historiques et littéraires, Paris, Michel Lévy frères, 1853.

Mérimée P., Romans et Nouvelles, H. Martineau (dir.), Paris, Gallimard, "Bibliothèque de la Pléiade », 1951.

Schmittlein R., Lokis, la dernière nouvelle de Prosper Mérimée, Bade, Éditions Art et Science, 1949.

\section{abstract}

\section{Prosper Mérimée and Poland}

Prosper Mérimée (1803-1870), writer and scholar, member of the French Academy, has often evoked in his correspondence, his fictional accounts and his historical studies, the political and cultural past of Poland as well as its conflicting relations with Russia during the 19th century. Unlike many of his romantic contemporaries, he was rather insensitive to the plight of the Poles and made no effort to understand them better. His fear of revolutionary convulsions, his anti-Catholic prejudices, his too superficial knowledge of local realities and his growing love for Russia throughout his life, probably explain his views. However, we should not forget his friendship with some Poles in exile in France and his support for the Historical and literary library of Paris.

\section{keywords}

Mérimée, Poland, Russia, anti-Russian revolts, Lithuania

\section{mots-clés}

Mérimée, Pologne, Russie, Révoltes antirusses, Lituanie 


\section{thierry laurent}

Thierry Laurent est docteur en littérature française médiévale, docteur en littérature française moderne et habilité à diriger des recherches. Ancien professeur de français langue étrangère à la Sorbonne, il est conférencier à I'université de Vilnius et chercheur au CRICES. II a publié une dizaine d'essais sur des romanciers français des XXe et XXle siècles, dont André Maurois, Albert Camus, Michel Déon et Patrick Modiano. II s'intéresse aussi beaucoup aux cultures slaves et baltes.

ORCID : https://orcid.org/0000-0003-0885-4433 